\title{
Plantations d'Atriplex canescens en vue de la restauration des parcours dégradés en steppe algérienne
}

\author{
Ouarda Amrani ${ }^{1 *}$ Abd Elmadjid Chehma ${ }^{2}$
}

\begin{abstract}
Mot-clés
Atriplex canescens, steppe, espace pastoral, gestion foncière durable, biomasse, composition chimique, surpâturage, Algérie
\end{abstract}

Submitted: 11 October 2017

Accepted: 20 April 2020

Published: 29 June 2020

DOI: $10.19182 /$ remvt.31879

\section{Résumé}

La steppe algérienne est le milieu d'élevage du mouton par l'exploitation de ses parcours. Dans sa partie aride, la vulnérabilité du milieu est aggravée par une exploitation principalement pastorale de plus en plus intense durant ces dernières décennies. Cette tendance régressive est caractérisée par une baisse de la productivité fourragère et les espèces pérennes sont les plus touchées. L'Etat algérien a mis en œuvre un programme d'aménagement des espaces steppiques très dégradés à travers la plantation d'espèces végétales résistantes et productives de biomasse comme Atriplex spp. L'objectif de ce travail a été d'estimer la productivité pastorale de quelques stations réhabilitées par la plantation d'Atriplex canescens, comparativement aux parcours naturels pâturés. Les résultats ont montré une amélioration du recouvrement végétal (de $49 \%$ à $60 \%$ ) et de meilleures valeurs pastorales (phytomasse, productivité, valeur énergétique et charge pastorale). Sur les zones réhabilitées une productivité énergétique et protéique de 604 unités fourragères lait / ha/an et 29 kg de matière sèche / ha/an, et une charge pastorale de 1,51 unité ovine / ha/an ont été enregistrées, alors que sur les parcours naturels dégradés ces valeurs ont été respectivement de 14 UFL, 0,422 kg MS/ha/an, et 0,03 unité ovine / ha/an. Les stations naturelles sont surexploitées et tendent vers un état de dégradation sévère de la végétation et des sols steppiques. A moins de restaurer ou de réhabiliter les parcours steppiques, leur capacité de productivité pastorale sera fortement réduite.

- Comment citer cet article : Amrani O., Chehma A.E., 2020. Plantation of Atriplex canescens for the restoration of degraded courses in the Algerian steppe. Rev. Elev. Med. Vet. Pays Trop., 73 (2): 113-121, doi: $10.19182 /$ remvt.31879

\section{INTRODUCTION}

En Algérie, les espaces steppiques sont menacés par la dégradation de leurs ressources naturelles. Le sol et le couvert végétal naturel pastoral notamment souffrent du surpâturage, du défrichement, de l'ensablement, de l'érosion éolienne du sol et de la végétation, ou de la salinisation. Ces évolutions écologiques constituent un sujet préoccupant pour les populations qui vivent de l'élevage (Nedjraoui et Bedrani, 2008; Ahmed, 2015).

L'élevage occupe cependant une place importante dans la politique agricole nationale (Bencherif, 2013 ; Yerou, 2013). Il joue un rôle

\footnotetext{
1. Département des sciences agronomiques, Faculté des sciences, Université Amar Thelédji, BP 37 Laghouat, Algérie.

2. Laboratoire de bio ressources sahariennes «Préservation et Valorisation », Faculté des sciences, Université Kasdi Merbah, 30000 Ouargla, Algérie.

* Auteur pour la correspondance

Email : mmehassinet@gmail.com
}

vital dans l'agriculture et l'économie du pays et représente une part substantielle dans le produit intérieur brut agricole (40\% selon Rekik et al., 2014). Néanmoins, il est confronté au problème de l'insuffisance fourragère (en quantité et qualité) et du changement des modes de conduite des animaux. Au niveau national, un bilan fourrager global réalisé à la fin des années 2000 a évalué le déficit fourrager à quatre milliards d'unités fourragères (UF) (Issolah, 2008). Ce problème de déficit fourrager avait déjà été signalé dans le passé pour l'ensemble de la steppe algérienne (Bourahla et Guittonneau, 1978), mais il s'est accentué.

La végétation naturelle et les sols de ces espaces s'avèrent dans un état de dégradation très avancé. Cette situation est liée à l'évolution des pratiques pastorales, aux pratiques de mise en valeur agricole (défrichements, labours, céréaliculture), à l'aridité du climat, à l'irrégularité des pluies, et à la longueur de la saison sèche (Aidoud et al., 2006). En particulier, le cheptel ovin a connu une croissance rapide. Il est passé de 18 millions à plus de 26 millions de têtes entre 2001 et 2014 selon les statistiques du ministère de l'Agriculture. Or, durant cette même période, la steppe a connu une longue période de déficit 
pluviométrique. Il en a résulté une pression accrue sur ces ressources. On estime que pendant ces années, la production de fourrage steppique a couvert moins de $30 \%$ des besoins du cheptel (Aidoud et al., 2006 ; Bencherif, 2013).

Le rabougrissement de la végétation pastorale et la diminution des surfaces steppiques accentuée par l'augmentation de la charge animale (Bensouiah, 2004) ont entraîné une détérioration du pouvoir de régénération de ces ressources. Pour réduire l'intensité du pâturage et de la dégradation qui en résulte, de nouveaux modes de gestion de la végétation pastorale ont été introduits dans les parcours steppiques en Algérie. Dans ce contexte, le Haut-Commissariat au développement de la steppe (HCDS) a procédé à la réhabilitation de ces parcours fortement dégradés par l'introduction de plantes et d'arbustes d'intérêt fourrager comme Atriplex spp. (A. canescens, A. nummularia, A. halimus), des luzernes (M. arborea, M. sativa) et Opuntia ficus indica. A. canescens, en particulier, a été choisi pour sa rapidité de croissance, sa résistance aux aléas climatiques, et sa valeur fourragère (Le Houérou, 2000 ; 2006).

Ces espaces à réhabiliter ont été réquisitionnés par les autorités locales qui sont devenues les seuls gestionnaires. Les bénéficiaires qui participent à ces projets deviennent plus conscients de l'intérêt de ces plantations et seraient prêts à les développer et à les préserver. Les actions opérationnelles comme la plantation d'espèces fourragères bénéficient aujourd'hui de l'adhésion des populations d'agropasteurs (MADR, 2007).

L'efficacité de ces nouveaux modes de pâturage mis en place par le HCDS a été évaluée par l'analyse de la composition floristique, et l'état de la végétation et de l'offre fourragère dans les parcours aménagés de 2008 à 2011 (Amghar et al., 2016) puis en 2012 (Salemkour et al., 2016). Ces actions ont montré l'amélioration de la richesse floristique et de la valeur pastorale dans les formations restaurées.

La wilaya de Laghouat, qui se situe dans l'étage bioclimatique semiaride au nord, et saharien au sud, représente $12 \%$ de la superficie totale de la région steppique algérienne. Cette wilaya agropastorale dispose d'un cheptel très important dominé par les ovins (plus de deux millions de têtes) dont l'effectif a triplé en une vingtaine d'années (Nedjraoui et Bédrani, 2008). Cependant, sous l'effet conjugué du surpâturage (augmentation de la charge pastorale) et de la sécheresse, des signes de dégradation des parcours sont apparus provoquant un déficit alimentaire pour le cheptel et obligeant les éleveurs à recourir aux compléments alimentaires (concentrés).

Dans cette étude, nous nous sommes intéressés aux parcours aménagés afin d'évaluer l'effet de la plantation d'arbustes fourragers sur la productivité pastorale des parcours. Nous avons tenté de répondre à la question suivante : la réhabilitation des parcours par plantation d'A. canescens, peut-elle apporter une amélioration significative des parcours steppiques dégradés, et si oui, comment mesurer cette amélioration? Pour y répondre, nous avons conduit des relevés de terrain dans le nord de Laghouat particulièrement concerné par les programmes d'aménagement nationaux.

\section{MATERIEL ET METHODES}

\section{Localisation et caractérisation des zones d'étude}

La wilaya de Laghouat occupe une position centrale, reliant les hauts plateaux avec le Sahara. Elle se situe à 400 kilomètres au sud de la capitale Alger ( $\left.33^{\circ} 48^{\prime} \mathrm{N}, 02^{\circ} 53^{\prime} \mathrm{E}\right)$ et couvre une superficie de 25052 kilomètres carrés (figure 1). C'est une zone de steppe regroupant trois ensembles géographiques présentant chacun des caractéristiques géomorphologiques et des formations végétales homogènes. Le premier ensemble est formé par les piémonts de l'Atlas saharien, zone à caractère agropastoral qui représente $30 \%$ de la superficie totale de la wilaya.

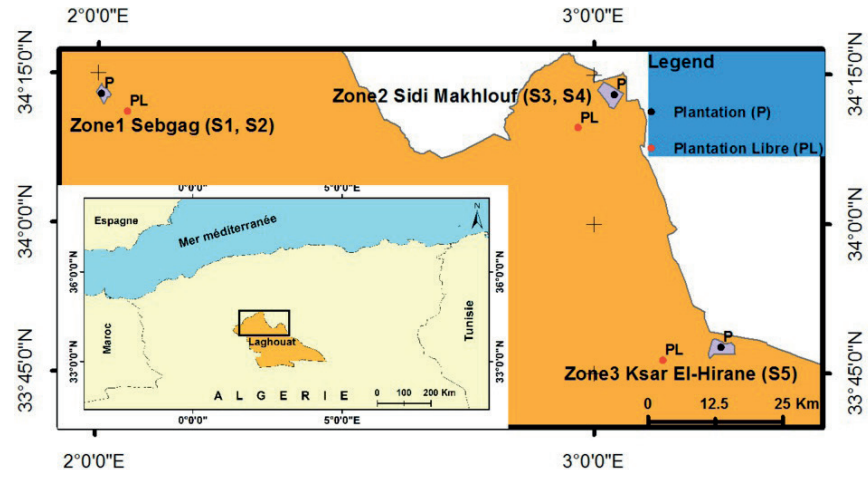

Figure 1 : les trois zones d'étude dans la wilaya de Laghouat en Algérie.

Ces piémonts sont formés principalement de vieux massifs forestiers et des pacages et parcours alfatiers. Le second ensemble est constitué par la plaine alluviale de l'Oued M'Zi et par un plateau à surface plane avec une charge caillouteuse en surface. Les sols y sont généralement peu profonds. Cette zone à caractère agropastoral représente plus de $60 \%$ de la superficie. Elle renferme de vastes étendues steppiques pour la plupart dégradées. Le dernier ensemble est une vaste étendue de parcours prédésertiques s'étalant vers le sud à base de chaméphytes peu appétées. Cet ensemble représente $10 \%$ de la superficie de la wilaya. Les terres de parcours occupent une superficie de 18429 kilomètres carrés soit $74 \%$ du territoire de la wilaya. Selon les services agricoles de la wilaya, les parcours en bon état, c'est-à-dire ceux dont la productivité fourragère est intéressante, ne représentent que $6 \%$ de la superficie totale des parcours de la wilaya. Les mêmes services évaluent à $68 \%$ les parcours dégradés et à $26 \%$ ceux qui sont aménagés.

Du point de vue climatique, la wilaya est caractérisée par un climat méditerranéen, semi-aride à saharien. Elle est répartie le long d'un gradient d'aridité entre les stations météorologiques d'Aflou et de Laghouat, avec respectivement $324 \mathrm{~mm}$ et $162 \mathrm{~mm}$ de pluviométrie moyenne annuelle pour la période 2004-2014 (tableau I). La répartition saisonnière des précipitations est marquée par la prédominance des pluies d'automne et de printemps $(29,52 \%$ et $29,41 \%)$ au nord. Au sud, c'est la saison automnale qui est la plus pluvieuse (55,48\%). Les données météorologiques recueillies entre les années 2005-2014 au niveau des deux stations météorologiques, nous ont permis d'établir les diagrammes ombrothermiques de Bagnouls et Gaussen (figure 2).

La durée et l'intensité de la saison sèche sont différentes d'une zone à l'autre, ce qui traduit une variabilité climatique spatiale dans la région. Le gradient pluviométrique décroît du nord vers le sud, et la période sèche dure plus de cinq mois au nord. D'autre part, elle couvre les douze mois au sud, qui est en déficit hydrique durant l'année entière.

\section{Tableau}

Caractéristiques et conditions climatiques de la wilaya de Laghouat en Algérie

\begin{tabular}{|c|c|c|}
\hline Station météorologique & Aflou & Laghouat \\
\hline Altitude (m) & 1400 & 750 \\
\hline $\begin{array}{l}\text { Précipitations moyennes (mm) } \\
\text { durant la période d'étude (2012-2014) }\end{array}$ & 361 & 125 \\
\hline Précipitations moyennes (mm) (2004-2014) & 324 & 162 \\
\hline Coefficient de variation de la pluviosité & 83 & 61 \\
\hline Température moyenne maximale $\left({ }^{\circ} \mathrm{C}\right)$ & 23,5 & 32,23 \\
\hline Température moyenne minimale $\left({ }^{\circ} \mathrm{C}\right)$ & 3,10 & 8,06 \\
\hline
\end{tabular}



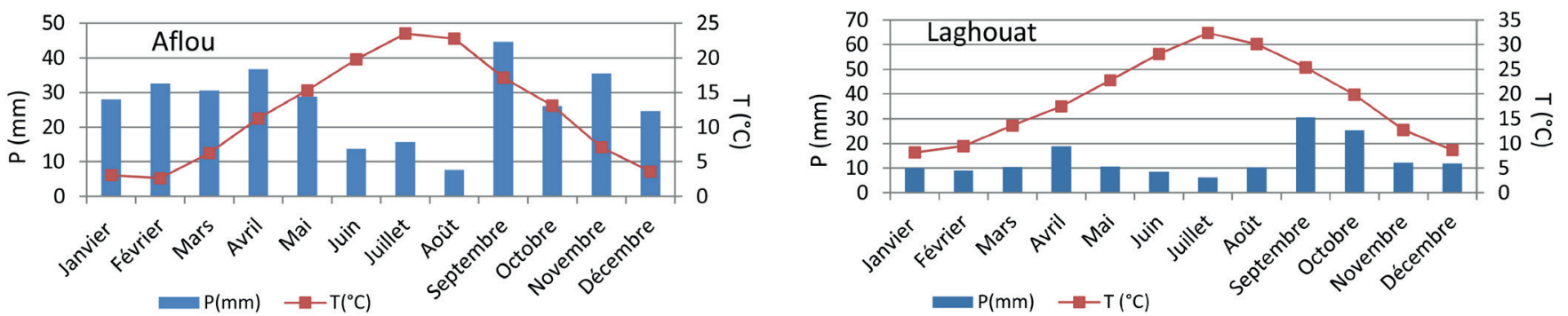

Figure 2 : diagramme ombrothermique de Bagnouls et Gaussen, stations météorologiques Aflou et Laghouat (2005-2014).

La pluviosité moyenne de la décennie 2005-2014 a présenté une grande variabilité interannuelle. En effet, l'année 2007 était la plus sèche à Aflou et à Laghouat (figure 3). La pluviosité moyenne annuelle a diminué de $17 \%$ à $27 \%$ dans les steppes algériennes durant le siècle dernier (Hammouda, 2009). La diminution générale des précipitations de la zone d'étude est comprise entre $7 \%$ à Aflou et $14 \%$ à Laghouat (Hammouda, 2009).

Laghouat présente une mosaïque pédologique dans laquelle cinq classes de sols sont distinguées : sols minéraux bruts, sols peu évolués, sols calcimagnésiques, sols isohumiques, et sols des dayas. Les sols dominants sont du type steppique, caractérisés par un horizon superficiel de faible profondeur (moins de $25 \mathrm{~cm}$ ), de texture sablolimoneuse à sablo-argileuse (Bneder, 2014).

Les plantations réalisées par le HCDS dans le Laghouat ont concerné les parcours dégradés présentant une couverture végétale pérenne inférieure à $20 \%$. Elles étaient constituées d'une plantation pure composée seulement d'A. canescens. Les plants d'A. canescens ayant servi au repeuplement des stations étudiées ont été élevés en pépinière durant trois mois avant d'être transplantés. Après plantation,

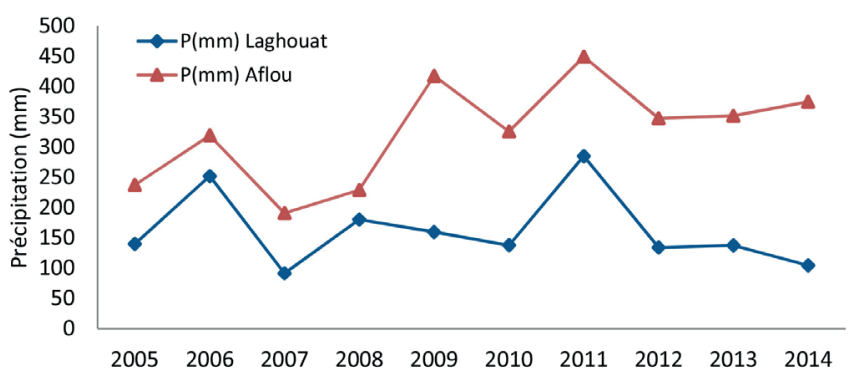

Figure 3 : variabilité interannuelle des pluies dans la wilaya de Laghouat en Algérie, stations météorologiques Aflou et Laghouat (2005-2014)

trois arrosages ont été effectués à raison de 20 litres par plant sur une période de deux mois. La densité moyenne des plantations a été estimée à 800 plants par hectare, avec un écartement inter et intra lignes de 5 mètres sur 2,5. Chaque zone a été mise en défens pendant les cinq années qui ont suivi sa plantation, leurs limites ont été matérialisées par des tumulus visibles de loin et leur protection a été assurée par un gardiennage.

\section{Sélection des stations}

L'étude a porté sur trois zones réparties selon un gradient est-ouest : Sebgag avec deux stations appartient au premier ensemble géographique, Sidi Makhlouf avec deux stations appartient au deuxième, et Ksar El Hirane avec une station appartient au troisième (figure 1). Dans ces trois zones, les plantations étaient âgées de cinq ans. Dans chacune des zones, deux stations ont été choisies : l'une aménagée et mise en défens depuis cinq ans, et l'autre conservée en parcours naturel soumis à un pâturage libre (figure 1). Dans la troisième zone, nous n'avons pas obtenu de résultat significatif pour le parcours en pâturage libre car il était quasiment nu. Nos résultats sont donc présentés pour cinq stations. Il faut noter que les stations en pâturage libre ne représentent pas à proprement parler des stations témoins. En effet, notre étude ne propose pas de comparer des parcelles réhabilitées avec des parcelles non réhabilitées, toutes choses égales par ailleurs. Il s'agit plutôt de comparer des parcelles « réhabilitées et mises en défens » avec des parcelles «non réhabilitées et en pâturage libre ». Le tableau II présente les caractéristiques des cinq stations. Ces caractéristiques soulignent le contexte particulièrement difficile de la zone de Ksar El Hirane constitué de microdunes de basse altitude soumis à un climat saharien.

L'objectif de l'évaluation était de comparer dans ces cinq stations l'état de recouvrement de la végétation, la phytomasse et la productivité pastorale. L'échantillonnage a été basé sur la méthode subjective (Gounot, 1969). Après une reconnaissance générale du territoire, qui

\section{Tableau II}

Présentation des stations dans la wilaya de Laghouat en Algérie

\begin{tabular}{|c|c|c|c|c|c|}
\hline \multirow{2}{*}{$\begin{array}{l}\text { Zone } \\
\text { Station }\end{array}$} & \multicolumn{2}{|c|}{ Sebgag } & \multicolumn{2}{|c|}{ Sidi Makhlouf } & \multirow{2}{*}{$\begin{array}{c}\text { Ksar El Hirane } \\
\text { S5 }\end{array}$} \\
\hline & S1 & S2 & S3 & S4 & \\
\hline Type d'aménagement & $\begin{array}{l}\text { Plantation } \\
\text { Mise en défens }\end{array}$ & $\begin{array}{l}\text { Parcours naturel } \\
\text { pâturé }\end{array}$ & $\begin{array}{l}\text { Plantation } \\
\text { Mise en défens }\end{array}$ & $\begin{array}{l}\text { Parcours naturel } \\
\text { pâturé }\end{array}$ & $\begin{array}{l}\text { Plantation } \\
\text { Mise en défens }\end{array}$ \\
\hline Superficie (ha) & 1600 & - & 1200 & - & 1100 \\
\hline Taux de réussite (\%) & 73 & - & 44 & - & 35 \\
\hline Altitude (m) & $<1500$ & $<1500$ & 900 & 900 & $<700$ \\
\hline Géomorphologie & $\begin{array}{l}\text { Glacis secondaire } \\
\text { ensablé }\end{array}$ & $\begin{array}{l}\text { Glacis secondaire } \\
\text { ensablé }\end{array}$ & $\begin{array}{l}\text { Glacis secondaire } \\
\text { ensablé }\end{array}$ & $\begin{array}{l}\text { Glacis secondaire } \\
\text { ensablé }\end{array}$ & Microdunes \\
\hline Etage bioclimatique & Semi-aride & Semi-aride & Saharien & Saharien & Saharien \\
\hline
\end{tabular}


a permis de sélectionner les stations selon des critères d'homogénéité (homogénéité des conditions écologiques, floristiques et physionomiques) (Gillet, 2000), des placettes de $100 \mathrm{~m}^{2}$ (10 m $\left.\times 10 \mathrm{~m}\right)$ ont été posées. Dans chaque zone, trois placettes ont été sélectionnées. Nous avons pris en considération des variables des stations, soit le type physionomique de la végétation notamment les espèces vivaces dominantes avec A. canescens.

\section{Echantillonnage du couvert végétal sur chaque station}

L'étude quantitative et qualitative a été effectuée selon la méthode des points quadrants linéaires (Daget et Poissonet, 1971). Elle a été utilisée pour évaluer l'état du couvert végétal en analysant les espèces présentes et leurs fréquences. La lecture a été faite tous les 10 centimètres le long d'un ruban gradué de 10 mètres tendu au-dessus de la végétation de chaque placette, soit sur 100 points de lecture par placette (Daget et Poissonet, 1971 ; Salemkour et al., 2016). Dans chaque station, le taux de recouvrement de la végétation (TRV) et la contribution spécifique des espèces ont été évalués. Au total, 360 relevés floristiques ont été effectués. Le recouvrement de la végétation est un paramètre quantitatif qui permet directement de distinguer les pâturages selon leur état actuel de dégradation. La contribution d'une espèce $i(\mathrm{CSi})$, par rapport à la somme des fréquences de l'ensemble des espèces, est assimilée à la probabilité de présence de cette espèce (Daget et Poissonet, 1971). La nomenclature botanique utilisée était celle de Quézel et Santa (1962).

\section{Relevés pédologiques}

Des relevés pédologiques ont accompagné les relevés floristiques. L'étude du sol était basée sur des échantillons prélevés sur une profondeur de 30 centimètres. Les paramètres pédologiques pris en compte étaient l'humidité, le $\mathrm{pH}$, la conductivité électrique, le taux de la matière organique et le taux du calcaire total. Les méthodes d'analyses utilisées étaient celles d'Aubert (1978).

\section{Estimation de la phytomasse et de la productivité des parcours}

La phytomasse aérienne de la strate herbacée des espèces pérennes et des arbustes d'un périmètre sylvopastoral est définie comme étant « la quantité de végétation (verte ou sèche) sur pied par unité de surface à un instant donné » (Aidoud, 1983), exprimée en kilogrammes de matière sèche (MS) par hectare. Il s'agit d'une mesure de la productivité des parcours. La méthode d'évaluation retenue était semi-destructive (Aidoud, 1983). Elle consistait pour chaque espèce à couper la partie aérienne de trois pieds de dimensions différentes (petite, moyenne et grande). La phytomasse aérienne a ensuite été calculée en multipliant le poids moyen en MS de chaque espèce par sa densité spatiale au sein de chaque placette (en g de MS/ha). Les valeurs moyennes sont ensuite calculées puis extrapolées (en $\mathrm{kg}$ de $\mathrm{MS} / \mathrm{ha}$ ).

\section{Composition chimique des fourrages}

La composition des fourrages a été déterminée par la méthode d'analyse chimique des fourrages (Marnay et al., 2014). L'analyse chimique a été réalisée sur les principales espèces pérennes présentes dans les différentes stations. Avant l'analyse, les échantillons des différentes stations ont fait l'objet de pesées à l'état frais. Une fois au laboratoire, ils ont été séchés à $85^{\circ} \mathrm{C}$ jusqu'à poids constant pour déterminer la matière sèche, broyés et conservés dans des boîtes hermétiques. A partir de ces échantillons, la matière organique (MO), la matière minérale (MM), la matière azotée totale (MAT) et la cellulose brute (CB) ont pu être déterminées. La valeur bromatologique des différentes espèces relevées a ensuite été déterminée en s'appuyant sur les méthodes officielles d'analyses appliquées en alimentation et en nutrition animale de l'Association of Official Analytical Chemist (AOAC) et de l'Association française de normalisation (Afnor).

\section{Production pastorale des parcours}

La production pastorale des parcours a été estimée en extrapolant sur une année les valeurs de la phytomasse des pérennes (productivité des parcours). Sur cette base, la production énergétique et la production azotée des fourrages pérennes ont été estimées. Ces valeurs ont été calculées à partir de la production de la biomasse des espèces qui les composent multipliée par leurs différentes valeurs nutritives (Chehma, 2005 ; Chehma et al., 2008).

\section{Charge pastorale théorique}

La capacité de charge théorique est définie par le nombre maximum d'herbivores qui peuvent pâturer une surface donnée, pendant un certain temps, sans détérioration de la végétation (Chehma, 2005). C'est le nombre moyen de têtes, pendant une période donnée, que peut supporter un pâturage. Les besoins énergétiques des animaux est en moyenne de 400 UF par an pour une brebis (Aidoud 1983). L'unité ovine est l'unité de référence permettant de calculer les besoins nutritionnels ou alimentaires d'une brebis et du troupeau (Aidoud 1983).

\section{Analyse statistique}

L'analyse de variance (Anova) et une analyse en composantes principales (ACP) ont été effectuées pour interpréter les résultats, avec le logiciel statistique XLSTAT (2009).

\section{RESULTATS}

L'analyse des données de couverture végétale a montré que les trois stations aménagées et mises en défens possédaient des TRV compris entre $49,4 \%$ et $60,5 \%$. Ces taux étaient très supérieurs à ceux des stations ouvertes au pâturage qui étaient compris entre $35 \%$ et $41 \%$ (figure 4). L'analyse de variance a révélé un effet significatif de la réhabilitation couplée à la mise en défens $(\mathrm{p}=0,0001)$ sur le TRV, qui s'est traduit par une augmentation importante des espèces pérennes.

Les résultats des analyses pédologiques (tableau III) indiquent, pour les trois zones, un $\mathrm{pH}$ alcalin à très alcalin. Les valeurs de conductivité électrique des sols ont souligné la différence entre les sols non salés de la zone de Sidi Makhlouf (S3) et les sols à faible salinité de Sebgag (S1) et de Ksar El Hirane (S5). Ces derniers étaient pauvres en matière organique, alors que ceux des deux autres zones avaient une teneur moyenne en MO (1,55 \% et 2,72)\%. Dans les trois zones, le taux de MAT a été très faible. La distribution spatiale du calcaire total était caractéristique des sols faiblement calcaires.

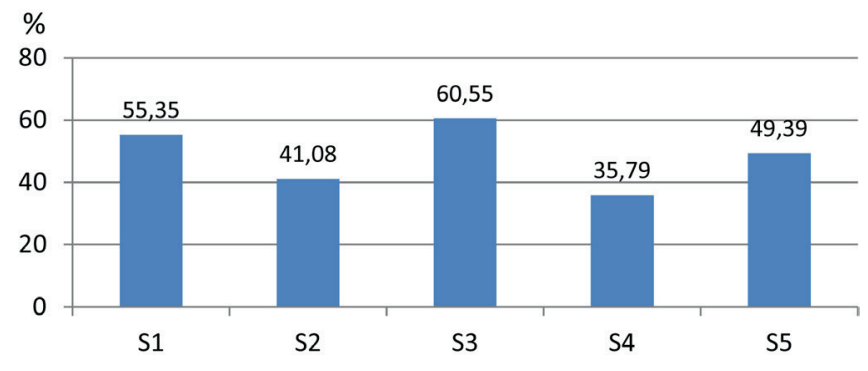

Figure 4 : taux de recouvrement végétal des stations étudiées dans la wilaya de Laghouat en Algérie (2012-2014). 


\section{Tableau III}

Paramètres morphologiques et analytiques des profils pédologiques des stations étudiées dans la wilaya de Laghouat en Algérie

\begin{tabular}{|c|c|c|c|}
\hline & Sebgag & $\begin{array}{c}\text { Sidi } \\
\text { Makhlouf }\end{array}$ & $\begin{array}{l}\text { Ksar El } \\
\text { Hirane }\end{array}$ \\
\hline Station & S1 & S3 & S5 \\
\hline $\mathrm{H}(\%)$ & 4,64 & 2,91 & 2,13 \\
\hline pH eau & 8,51 & 8,38 & 8,64 \\
\hline $\begin{array}{l}\text { Conductivité électrique } \\
\text { à } 25^{\circ} \mathrm{C}(\mathrm{mmho} / \mathrm{cm})\end{array}$ & 0,253 & 0,194 & 0,292 \\
\hline Matière sèche (\%) & 95,36 & 97,09 & 97,87 \\
\hline Matière minérale (\%) & 98,45 & 97,25 & 99,3 \\
\hline Matière organique (\%) & 1,55 & 2,72 & 0,7 \\
\hline Matière azotée (\%) & 0,028 & 0,024 & 0,021 \\
\hline Taux de calcaire (\%) & 2,56 & 3,51 & 3,36 \\
\hline
\end{tabular}

Les familles les mieux représentées dans les trois zones ont été les Asteraceae (22 \%), les Fabaceae (14,71 \%) et les Poaceae (8,82 \%). Dans les stations aménagées, $A$. canescens était l'espèce pérenne dominante et représentait entre $22,4 \%$ et $48,8 \%$ de la phytomasse présente. Dans les stations naturelles ouvertes au pâturage, peu d'espèces pérennes étaient présentes. En particulier, sur la station naturelle ouverte au pâturage de Sebgag, seules deux espèces pérennes étaient présentes (tableau IV). Nos relevés soulignent aussi la réapparition de l'alfa (Stipa tenacissima), une espèce qui avait fortement régressé dans les premières années qui avaient suivi les aménagements. Dans les stations aménagées de Sebgag (S1) et de Sidi Makhlouf (S3), cette espèce a contribué pour $10 \%$ à $12 \%$ de la phytomasse.

Le test Anova appliqué aux moyennes annuelles des compositions chimiques des espèces pérennes (moyenne sur les quatre saisons) a révélé que l'aménagement a eu un impact positif $(\mathrm{p} \leq 0,01)$ sur la MAT des espèces pérennes. Les espèces pérennes étudiées étaient moyennement riches en fibres brutes (taux de cellulose brute compris entre $28 \%$ et $35 \%$ de la MS). Les valeurs des taux en MM oscillaient de 7,59 \% à 16,45\% de la MS dans les stations aménagées, et de $3,15 \%$ à 4,76 \% dans les stations ouvertes au pâturage, durant les deux années de l'étude (tableau V).

Létude des potentialités des espèces pérennes dans les différentes stations a révélé que les parcours aménagés et protégés présentaient des valeurs meilleures de productivités pastorales que les parcours naturels ouverts au pâturage (tableau VI). Les stations aménagées ont présenté des valeurs élevées en phytomasse, avec un maximum de $1291 \mathrm{~kg} \mathrm{MS} / \mathrm{ha}$ et un minimum de $1168 \mathrm{~kg} \mathrm{MS} / \mathrm{ha}$. En revanche, les stations ouvertes au pâturage libre ont eu des valeurs de phytomasse comprises entre 12,38 et 20,62 kg MS/ha. La productivité fourragère en terme de phytomasse a donc été augmentée dans les stations aménagées de 10 à 50 fois par rapport aux stations naturelles.

La production pastorale moyenne annuelle a aussi été la plus élevée dans les stations aménagées de Sebgag et de Sidi Makhlouf, marquées par la présence d'Atriplex et d'alfa, avec respectivement 278,56 et $263,65 \mathrm{~kg}$ de $\mathrm{MS} / \mathrm{ha} / \mathrm{an}$. Elle a été beaucoup plus faible dans la zone aménagée de Ksar El Hirane dominé, en plus d'Atriplex, par Anabasis articulata (179,61 kg de MS/ha/an). Ces valeurs ont représenté entre 3 et 15 fois plus de productions pastorales que les stations non réhabilitées ouvertes au pâturage.

Le tableau VI montre les résultats des valeurs énergétiques moyennes annuelles en unité fourragère lait (UFL) et en unité fourragère viande (UFV). Les meilleures valeurs de la production énergétique ont été enregistrées dans les zones aménagées. Ces valeurs étaient entre 20 et 30 fois supérieures aux valeurs de la production énergétique des stations non réhabilitées ouvertes au pâturage libre. Les valeurs énergétiques des productions fourragères ont été particulièrement bonnes dans la station aménagée de Ksar El Hirane (450 UFV/ha).

Les stations aménagées ont présenté aussi les valeurs azotées les plus importantes avec des valeurs comprises entre 24,7 et $29,47 \mathrm{~kg} / \mathrm{ha}$ pour les protéines digestibles dans l'intestin grêle limitées par l'azote (PDIN), et entre 52,46 et $60,18 \mathrm{~kg} /$ ha pour les protéines digestibles dans l'intestin grêle limitées par l'énergie (PDIE). Ces valeurs ont été de 10 à 58 fois supérieures à celles des stations non réhabilitées ouvertes au pâturage libre.

\section{Tableau IV}

Contribution spécifique des espèces pérennes des stations étudiées dans la wilaya de Laghouat en Algérie

\begin{tabular}{|c|c|c|c|c|c|}
\hline \multirow[b]{2}{*}{ Station } & \multicolumn{2}{|c|}{ Sebgag } & \multicolumn{2}{|c|}{ Sidi Makhlouf } & \multirow{2}{*}{$\begin{array}{c}\text { Ksar El Hirane } \\
\text { S5 }\end{array}$} \\
\hline & S1 & S2 & S3 & S4 & \\
\hline Retama raetam & - & - & 3,96 & - & 6,65 \\
\hline Thymelaea microphylla & - & - & 3,48 & 11,47 & 17,75 \\
\hline Helianthemum lippii & - & - & 2,15 & 0 & 2,37 \\
\hline Atractylis serratuloides & - & - & 7,85 & 10,71 & 0,35 \\
\hline Stipa tenacissima & 12,73 & 29,47 & 10,24 & 13,39 & - \\
\hline Pituranthos sp. & - & - & 2,05 & - & - \\
\hline Artemisia campestris & 13,92 & 38,82 & 1,53 & 5,42 & - \\
\hline Atriplex canescens & 49,6 & - & 27,6 & - & 22,4 \\
\hline Stipa parviflora & 5,21 & - & 4,47 & 2,38 & - \\
\hline Lygeum spartum & 7,97 & - & 4,78 & - & - \\
\hline Anabasis articulata & - & - & 5,02 & - & 31,67 \\
\hline Astragalus armatus & - & - & 10,81 & 24,2 & \\
\hline Salsola vermiculata & - & - & 7,09 & 6,73 & 3,89 \\
\hline Peganum harmala & - & - & - & - & 4,75 \\
\hline
\end{tabular}

S2 et S4 : stations non réhabilitées 


\section{Tableau V}

Caractéristiques de la composition chimique des espèces pérennes des stations étudiées dans la wilaya de Laghouat en Algérie

\begin{tabular}{|c|c|c|c|c|c|}
\hline \multirow[b]{2}{*}{ Station } & \multicolumn{2}{|c|}{ Sebgag } & \multicolumn{2}{|c|}{ Sidi Makhlouf } & \multirow{2}{*}{$\begin{array}{c}\text { Ksar El Hirane } \\
\text { S5 }\end{array}$} \\
\hline & S1 & S2 & S3 & S4 & \\
\hline Matière sèche (\%) & 65 & 70 & 64 & 68 & 58 \\
\hline Matière azotée totale (\% MS) & 3,02 & 2,57 & 3,06 & 2,74 & 3,87 \\
\hline Matière minérale (\% MS) & 9,59 & 4,76 & 7,59 & 3,15 & 16,45 \\
\hline Matière organique (\% MS) & 90,41 & 95,24 & 87,41 & 97,64 & 83,55 \\
\hline Cellulose brute (\% MS) & 35,20 & 32,50 & 32,50 & 33,00 & 28,80 \\
\hline
\end{tabular}

S2 et S4 : stations non réhabilitées; MS : matière sèche

Tableau VI

Performances pastorales annuelles des stations étudiées dans la wilaya de Laghouat en Algérie

\begin{tabular}{|c|c|c|c|c|c|c|}
\hline \multirow{2}{*}{ Station } & & \multicolumn{2}{|c|}{ Sebgag } & \multicolumn{2}{|c|}{ Sidi Makhlouf } & \multirow{2}{*}{$\begin{array}{c}\text { Ksar El Hirane } \\
\text { S5 }\end{array}$} \\
\hline & & S1 & S2 & S3 & S4 & \\
\hline \multicolumn{2}{|c|}{ Phytomasse des pérennes (kg MS /ha) } & 1265,88 & 12,38 & 1168,37 & 20,62 & 1290,57 \\
\hline \multicolumn{2}{|c|}{ Production pastorale (kg MS/ha) } & 278,56 & 90,40 & 263,65 & 11,93 & 179,61 \\
\hline \multirow[t]{2}{*}{ Production énergétique } & UFL/ha & 604,15 & 64,02 & 523,77 & 14,37 & 563,63 \\
\hline & UFV/ha & 232,22 & 50,55 & 407,30 & 11,43 & 450,38 \\
\hline \multirow[t]{2}{*}{ Production azotée } & PDIN kg/ha & 29,47 & 1,64 & 24,70 & 0,42 & 25,24 \\
\hline & PDIE kg/ha & 60,18 & 5,77 & 52,46 & 1,31 & 54,69 \\
\hline \multicolumn{2}{|c|}{ Charge pastorale théorique ( U. ovine /ha/ an) } & 1,51 & 0,16 & 1,30 & 0,03 & 1,41 \\
\hline
\end{tabular}

S2 et S4 : stations non réhabilitées ; UFL : unité fourragère lait ; UFV : unité fourragère viande ; PDIE : protéines digestibles dans l'intestin grêle limitées par l'énergie ; PDIN : protéines digestibles dans l'intestin grêle limitées par l'azote

Le calcul de la charge pastorale théorique (400 UF/ha/an) a montré que dans les stations plantées et mises en défens (tableau VI) elle pouvait atteindre 1,30 à 1,51 unité ovine / ha/an. En revanche, dans les stations naturelles et ouvertes au pâturage, la charge théorique était très faible, entre 0,03 et 0,16 unité ovine/ha/an, ce qui nécessitait 6 à 33 hectares pour subvenir au besoin d'une seule unité ovine. Au total, les charges pastorales théoriques des stations réhabilitées de Sebgag et de Sidi Makhlouf ont été respectivement 9,4 à 43 fois supérieures à celles des stations non réhabilitées et ouvertes au pâturage.

Pour différencier les différentes stations entre elles sur une base multivariée, nous avons réalisé une ACP. Les variables actives utilisées ont été le taux de MS, le TRV, les valeurs énergétiques (UFV), la productivité pastorale (phytomasse) et les précipitations. L'axe 1 retenait $65,49 \%$ de l'inertie totale, l'axe 2 retenait $27,71 \%$ de l'inertie, ce qui a conduit au très bon taux d'inertie expliquée de 93,2\%.

La projection des variables est bien présentée sur le cercle de corrélations (figure 5) qui montre les relations existantes entre elles. Il y a eu une forte corrélation entre la productivité pastorale et le TRV, une corrélation inverse mais forte entre la production énergétique (UFV) et la production de MS. Les précipitations et la MS ont été fortement corrélées. L'axe 1 opposait un premier groupe formé par les variables, soit le TRV, l'énergie (UFV) et la productivité pastorale qui étaient corrélés positivement entre eux, et un deuxième groupe formé par le taux de MS. L'axe 2 était formé par la variable précipitations.

L'ACP a montré que la productivité dans les stations aménagées a été moins liée aux précipitations que dans les stations naturelles (figure 5). Les stations plantées et mises en défens, qui bénéficiaient d'une plus grande biomasse pérenne, ont constitué des écosystèmes plus résistants à l'aridité que les stations sans plantation. La plantation
d'Atriplex a ainsi constitué un moyen d'augmenter la résilience de l'écosystème, en plus de constituer une réserve fourragère (Kessler, 1990). La projection des cinq zones sur le graphe de l'ACP a montré un impact très net des aménagements (stations S1, S3 et S5). La synthèse des données par l'ACP a souligné que l'amélioration des

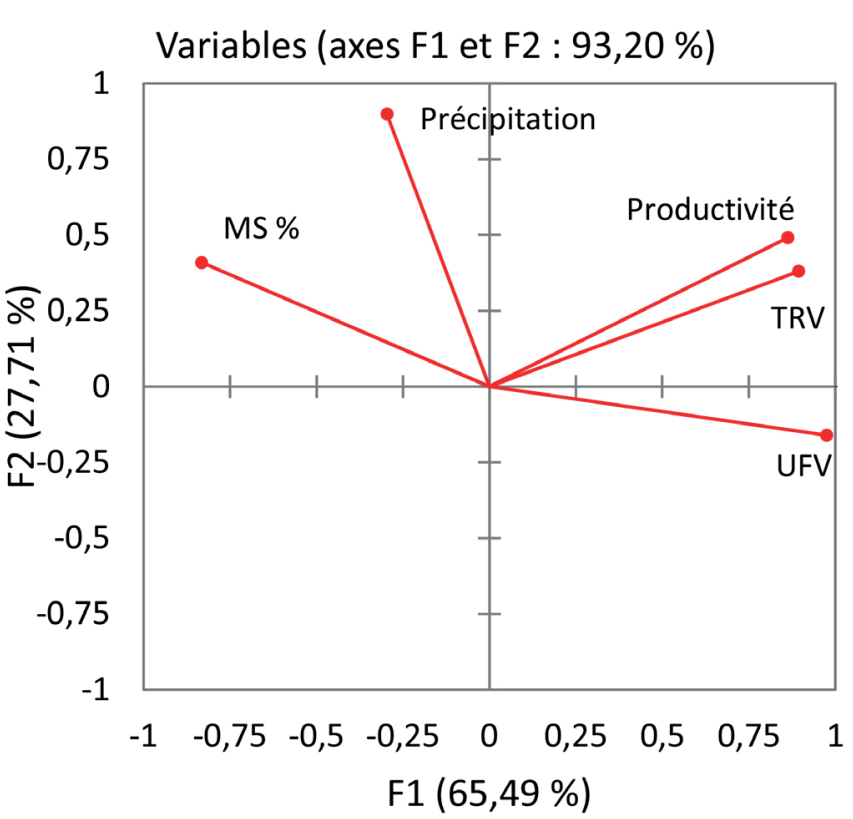

Figure 5 : cercle de corrélation des variables ( $A C P$ sur la valeur pastorale et précipitation), wilaya de Laghouat en Algérie (20122014). MS : matière sèche; TRV : taux de recouvrement végétal. 
caractéristiques productives dans les stations plantées en Atriplex (TRV, phytomasse, diversité floristique) s'est répercutée, d'une part, sur l'augmentation quantitative de la production pastorale et, d'autre part, sur la valeur alimentaire des fourrages.

La projection des stations sur le plan factoriel 1-2 de l'ACP (figure 6) a révélé trois groupes : le premier groupe comprenait les stations aménagées de Segbag (S1) et de Sidi Makhlouf (S3) qui étaient les deux stations les plus productives et qui possédaient une bonne couverture végétale. Le deuxième groupe renfermait la station aménagée de Ksar El Hirane (S5) qui était moins productive mais présentait une valeur énergétique importante. Le troisième groupe renfermait les deux stations non réhabilitées et ouvertes au pâturage (S2 et S4) dans lesquelles la productivité moyenne en MS par hectare restait très dépendante des précipitations qui demeuraient le principal facteur limitant pour la production d'unités fourragères.

Dans les stations aménagées de Sebgag et de Sidi Makhlouf, l'amélioration de la production fourragère provenait en grande partie de la biomasse produite par A. canescens. A Segbag, cet apport de biomasse issu d'Atriplex a été doublé par un regain d'Artemisia campestris et d'alfa, tandis qu'à Sidi Makhlouf, nous avons noté une diversité floristique beaucoup plus grande. Dans la station aménagée de Ksar El Hirane, c'était la dominance d'Anabasis articulata et, dans une moindre mesure, d'A. canescens et de Thymelaea microphylla qui ont expliqué le regain de la production pastorale.

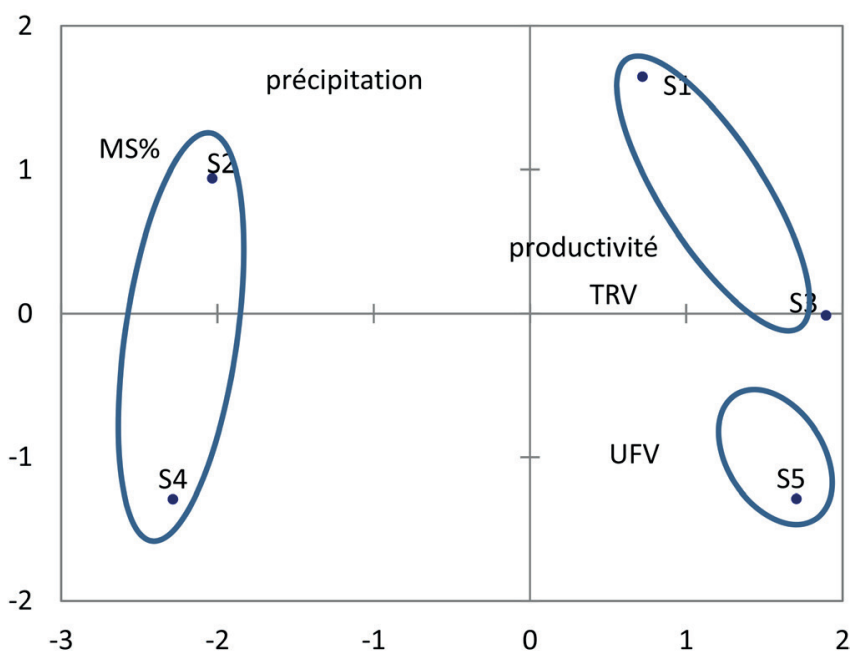

Figure 6 : répartition des stations de Laghouat en Algérie sur le plan factoriel 1-2 de I'ACP (axe $1=65,49 \%$ d'inertie, axe 2 $=27,71 \%$ ). MS matière sèche ; TRV : taux de recouvrement végétal ; UFV : unité fourragère lait ; S1, S2 : stations de Sebgag ; S3, S4 : stations de Sidi Makhlouf, S5 : station de Ksar El Hirane; S2 et $S 4$ : stations non réhabilitées.

\section{DISCUSSION}

L'objectif de ce travail était d'évaluer dans quelle mesure la réhabilitation par la plantation d'A. canescens et la mise en défens amélioraient la productivité pastorale des parcours dégradés. Ce travail a consisté à estimer le taux de recouvrement de la végétation, la diversité floristique, la productivité pastorale des parcours, la valeur bromatologique fourragère et protéique des espèces pérennes présentes dans les stations réhabilitées, et dans les stations non réhabilitées et ouvertes au pâturage. Les résultats ont montré que la réhabilitation a provoqué des effets importants sur tous ces paramètres.

Dans les stations ayant bénéficié des replantations et des mises en défens, un recouvrement global de la végétation et une diversité floristique très supérieurs à ceux des stations non aménagées et ouvertes au pâturage ont été observés. Amghar et al. (2016) indiquent que le TRV est plus élevé dans les zones avec plantation d'Atriplex que dans des zones mises en défens mais non plantées (respectivement $75,2 \%$ et 67,67 \%). Ces résultats sont aussi cohérents avec les travaux conduits au Maroc par Hachmi et al. (2015) qui mentionnent des TRV différents entre des parcours plantés d'A. nummularia (51\%), des mises en repos (24\%) et des travaux de conservation de l'eau et du sol (17\%).

L'amélioration du TRV des stations aménagées était due à $A$. canescens qui a créé une couverture végétale permanente et a contribué au processus de remontée biologique, augmentant le taux de recouvrement permanent de la biomasse pérenne, ce qui est là aussi cohérent avec la littérature (Le Houérou, 1995 ; Amghar et al., 2016). Le couvert végétal, plus élevé dans les stations aménagées depuis cinq ans, témoignait d'une régénération des pérennes ce qui représente un rempart contre la dégradation dans les parcours.

Les propriétés nutritives d'une espèce varient selon les saisons et les écotypes. De même, la contribution spécifique tient compte aussi bien des espèces fourragères que des espèces non fourragères (Akpo et al., 2002). La réhabilitation effectuée dans le Sud tunisien (Le Floc'h et al., 1999) a permis de reconstituer une steppe très dégradée et un certain nombre d'espèces ont pu être réinstallées (Stipa parviflora, Artemisia campestris).

Il y a eu une grande variabilité des composants chimiques entre les différentes espèces analysées, qui serait liée au milieu, à la saison et à la variabilité génétique entre espèces (Chehma et al., 2008). La productivité moyenne en matière sèche par hectare a été très dépendante des précipitations pour les stations non aménagées, un facteur limitant pour la production des unités fourragères.

La réhabilitation a permis au cortège floristique de se reconstituer et de produire une nouvelle biomasse. Selon Le Houérou (1995), environ $80 \%$ de la biomasse des steppes est assurée par les espèces pérennes. $A$. canescens, espèce adaptée aux différentes contraintes météorologiques et pédologiques de la zone steppique (Le Houérou, 2000), a permis la création d'un nouveau cortège floristique.

Selon la tranche pluviométrique annuelle (105-256 mm), la production de biomasse sèche reste très hétérogène et oscille entre 520 et $1220 \mathrm{~kg}$ / ha (Yerou, 2013). Dans une steppe aride en Syrie, Louhaichi et al. (2012) rapportent une production de phytomasse de $490 \mathrm{~kg} / \mathrm{ha}$ par contre $2340 \mathrm{~kg} / \mathrm{ha}$ en zones protégées. Cependant, Hachmi et al. (2015) notent $2600 \mathrm{~kg} / \mathrm{ha}$ pour une zone réhabilitée par plantation d'Atriplex dans une steppe aride au Maroc. Par ailleurs, les valeurs de phytomasse totale obtenues dans les zones aménagées de notre étude ont été inférieures à celles rapportées par Louhaichi et al. (2012), et Hachmi et al. (2015), car la phytomasse des espèces annuelles n'a pas été prise en considération.

Aidoud (1989) montre que les parcours se dégradent fortement lorsque la charge pastorale potentielle est (environ 8 ha / unité ovine) supérieure à la charge réelle des parcours, ce qui donne lieu à un surpâturage intense. La charge qui permet l'équilibre a été estimée en 1982 à 0,25 tête/ha, soit 4 hectares pour un ovin, alors que celle constatée à Djebel Amour est quatre fois plus élevée, atteignant 0,96 tête/ha (Bensouiah, 2004). Elle est même beaucoup plus élevée dans les zones les plus accessibles, comme dans la commune d'Ain Sidi Ali qui présente une charge 11 fois supérieure à la norme citée (Bensouiah, 2004). Cette surcharge animale, avec une phytomasse faible par rapport à leur potentialité et un pâturage sans temps de repos suffisant, ne permet pas d'assurer une régénération du couvert végétal. Cette situation est à l'origine de la dégradation de nombreux parcours (Bencherif, 2013). L'influence des précipitations n'est pas écartée ; Allreda et al. (2014) mentionnent son effet sur la productivité, et le bilan entre les précipitations et l'évaporation est souvent négatif (Aidoud et al., 2006). 
La différence de la valeur énergétique entre les stations aménagées et les stations ouvertes au pâturage serait due principalement à la pression de pâturage exercée dans les stations ouvertes au pâturage. Cette pression diminue leur productivité pastorale. En revanche, les stations aménagées jouent un rôle fondamental dans l'augmentation de la productivité pastorale : A. canescens a amélioré les disponibilités fourragères des parcours en zones steppiques à faible pluviométrie de Laghouat comme cela a été reporté dans d'autres wilayas. La production pastorale est significativement corrélée au couvert végétal mesuré (Aidoud, 1989).

Les plantations d'Atriplex peuvent être utilisées comme une réserve fourragère en été et en hiver, comblant la carence de fourrage qui se manifeste avant la croissance printanière des espèces fourragères herbacées (Kessler, 1990) et permet au bétail de supporter de longues périodes de carence alimentaire dues à la sécheresse. Elle sert, selon Brouri (2011), à améliorer la productivité pastorale de 30-50 UF/ha à plus de $600 \mathrm{UF} / \mathrm{h}$. Elle contribue aussi à protéger les sols et bien valoriser les espaces dégradés, même ceux les plus improductifs, comme les zones dunaires, salées, hamadas et terrains accidentés, à modifier la composition floristique, ainsi qu'à augmenter la diversité et le taux de recouvrement végétal. De nombreux exemples au Maghreb ont été rapportés par Acherkouk et El Houmaizi (2013).

Ces arbustes ont permis l'augmentation de la productivité pastorale dans la région d'étude qui avait une forte intensité de charge, ce qui a représenté l'une des stratégies efficaces pour la réduction du risque d'érosion lié aux conditions de dégradation par le surpâturage. Malgré la sécheresse et l'aridité du climat, cet aménagement a produit un impact positif sur la végétation (diverses espèces fourragères importantes sont réapparues), en améliorant le recouvrement végétal et la production fourragère en matière sèche et en énergie. La technique de plantation d'arbustes fourragers représente la meilleure technique en terme de restauration (Hachmi et al., 2015).

\section{- CONCLUSION}

L'utilisation de la productivité pastorale représente un outil précieux dans la gestion des ressources pastorales des parcours steppiques, permettant à la fois de répondre aux besoins de l'éleveur et à la protection de cet écosystème fragile (Rekik, 2014). La végétation steppique, dont la qualité fourragère est valorisée par la conduite de troupeaux ovins au pâturage, est soumise à la pression du surpâturage et de la sécheresse. La plantation d'A. canescens permet l'augmentation de la production de biomasse et la régénération des espèces végétales pérennes, et par conséquent la reconstitution des parcours steppiques. La qualité bromatologique des parcours et la densité de recouvrement sont fortement améliorées par ces plantations, lorsqu'elles sont doublées d'une mise en défens.

La productivité pastorale différenciée entre des stations réhabilitées et des stations non réhabilitées ouvertes au pâturage peut constituer $a$ priori un meilleur indicateur que la diversité de liste floristique globale de la valeur pastorale, car les espèces pérennes sont moins corrélées aux aléas climatiques que les espèces annuelles. Nos résultats ont confirmé que, dans le cas des steppes de Laghouat, la réhabilitation par la reconstitution des pâturages dégradés est permise par des actions d'aménagement pastoral basées sur les plantations d'Atriplex. Cela a pour incidence en particulier : a) l'augmentation du recouvrement végétal qui est une conséquence de l'augmentation de l'offre fourragère ; la phytomasse des pérennes a été 10 à 56 fois plus importante dans les parcours aménagés et mis en défens que dans les parcours naturels ouverts au pâturage ; b) l'augmentation de la valeur fourragère énergétique des parcours aménagés et mis en défens qui a été 9 à 22 fois supérieure à celle des parcours naturels ouverts au pâturage ; c) l'augmentation de la valeur protéique des parcours aménagés et mis en défens qui a été 18 à 58 fois supérieure à celle des parcours naturels ouverts au pâturage ; d) la charge pastorale théorique ovine des stations aménagées qui a été multipliée par 9,4 à 43 fois ; et e) la productivité dans les stations aménagées qui a été moins influencée par les précipitations.

Ces résultats mettront à la disposition des opérateurs sur le terrain (éleveurs, développeurs, autorités locales) des outils pour une gestion rationnelle des parcours aménagés, ainsi que des indicateurs d'évolution de ces espaces aidant à la prise de décisions futures.

\section{Remerciements}

Les auteurs adressent leurs sincères remerciements aux responsables du Haut-Commissariat au développement de la steppe pour leur collaboration pendant cette étude.

\section{Déclaration des contributions des auteurs}

OA et $\mathrm{AEC}$ ont participé à la conception et à la planification de l'étude ; OA a recueilli les données, a collecté les échantillons sur le terrain, a mis en œuvre les analyses au laboratoire, a effectué les analyses statistiques et a rédigé le manuscrit ; $\mathrm{AEC}$ a révisé le manuscrit.

\section{Conflits d'intérêts}

Les auteurs affirment qu'il n'existe aucun conflit d'intérêts.

\section{REFERENCES}

Acherkouk M. et El Houmaizi M.-A., 2013. Evaluation de l'impact des aménagements pastoraux sur la dynamique de la production des pâturages dégradés au Maroc oriental. Ecol. Mediterr., 39 : 69-84, doi : 10.3406/ ecmed.2013.1281

Ahmed Z., 2015. Determination and analysis of desertification process with satellite data Asat-1 and Landsat in the Algerian steppe. Eng. Geol. Soc. Territory, 2: 1847-1852, doi: 10.1007/978-3-319-09057-3_327

Aidoud A., 1983. Contribution à l'étude des écosystèmes steppiques du sud oranais. Thèse $3^{\mathrm{e}}$ cycle, USTHB, Alger, Algérie, 255 p.

Aidoud A., 1989. Contribution à l'étude des écosystèmes pâturés des hautes plaines Algéro-oranaises. Fonctionnement, évaluation, et évolution des ressources végétales. Thèse Doct. USTHB, Alger, Algérie, 240 p.

Aidoud A., Le Floc'h E., Le Houérou H.-N., 2006. Les steppes arides du nord de l'Afrique. Sécheresse, 17 (1-2) : 19-30

Akpo L.-E., Masse D., Grouzis M., 2002. Length of fallow period and pastoral value of herbaceous plants in the Sudanese zone of Senegal. Rev. Elev. Med. Vet. Pays Trop., 55 (4): 275-283, doi: 10.19182/remvt.9815

Allred B.-W., Scasta J.-D., Hovickb T.-J., Fuhlendorf S.-D., Hamilton R.G., 2014. Spatial heterogeneity stabilizes livestock productivity in a changing climate. Agric. Ecosyst. Environ., 193: 37-41, doi: 10.1016/j. agee.2014.04.020

Amghar F., Langlois E., Forey E., Margerie P., 2016. La mise en défens et la plantation fourragère : deux modes de restauration pour améliorer la végétation, la fertilité et l'état de la surface du sol dans les parcours arides algériens. BASE, 20 (3) : 386-396, doi: 10.25518/1780-4507.12576

Aubert G., 1978. Méthodes d'analyses des sols. CHADR, Marseille, France, $188 \mathrm{p}$.

Bencherif S., 2013. L'élevage agropastoral de la steppe algérienne dans la tourmente : enquêtes et perspectives de développement. Mondes Dév., 1 (161) : 93-106, doi: 10.3917/med.161.0093

Bensouiah R., 2004. Pasteurs et agro-pasteurs de la steppe algérienne. Strates, 11

BNEDER, 2014. Analyse de l'aptitude à la culture de l'olivier des sols de la région de Laghouat. BNEDER, Alger, Algérie, 202 p.

Bourahla A., Guittonneau G., 1978. Nouvelles régénération des nappes alfatières en liaison contre la désertification. Bull. Inst. Ecol. Appl., 1 : 19-40

Brouri L., 2011. Impacts des changements climatiques sur la gestion durable des ressources pastorales et des parcours dans les zones arides et semiarides de I'Algérie. In : Atelier sous régional sur l'effet du changement climatique sur l'élevage et la gestion durable des parcours dans les zones arides et semi-arides du Maghreb, Ouargla Algérie, 21-24 nov. 2011, 29-38

Chehma A., 2005. Etude floristique et nutritive spatio-temporelle des parcours camelins du Sahara septentrional algérien. Cas des régions de Ouargla et Ghardaia. Thèse Doct., Université d'Annaba, Algérie, 178 p. 
Chehma A., Faye B., Djebbar M.-R., 2008. Productivité fourragère et capacité de charge des parcours camelins du Sahara septentrional algérien. Sécheresse, 19 (2) : 115-121

Daget P., Poissonet J., 1971. Une méthode d'analyse phycologiques des prairies. Ann. Agron., 22 (1) : 5-41

Gillet F., 2000. La phytosociologie synusiale intégrée. Guide méthodologique. Documents du laboratoire d'écologie végétale et de phytosociologie, $1^{\text {re }}$ édn. Université de Neuchâtel / Institut de botanique, Suisse, 68 p.

Gounot M., 1969. Méthode d'étude quantitative de la végétation. Masson, Paris, France, $314 \mathrm{p}$.

Hachmi A., El Alaoui F.-E., Acherkouk M., Mahyou H., 2015. Parcours arides du Maroc : restauration par mise en repos, plantations pastorales et conservation de l'eau et du sol. Geo-Eco-Trop., 39 (2) : 185-204

Hammouda R., 2009. Contribution à I'élaboration d'un modèle de gestion durable d'un parcours steppique dans la commune de Hadj Mechri Wilaya de Laghouat. Thèse Magister, USTHB, Alger, Algérie, 142 p.

Issolah R., 2008. Les fourrages en Algérie : Situation et perspectives de développement et d'amélioration. Rech. Agron., 22 : 34-47

Kessler J.-J., 1990. Atriplex forage as a dry season supplementation feed for sheep in the Montane Plains of the Yemen Arab Republic. J. Arid Env., 19: 225-234, doi: 10.1016/S0140-1963(18)30821-8

Le Floc'h E., Neffati M., Chaieb M., Floret C., Pontanier R.,1999. Rehabilitation experiment at Menzel Habib, Southern Tunisia. Arid Soil Res. Rehabil., 13: 357-68, doi: 10.1080/089030699263249

Le Houérou H.-N., 1995. Dégradation, régénération et mise en valeur des terres sèches d'Afrique du Nord. Orstom, Montpellier, France, 53 p. (Coll. L’homme peut-il faire ce qu'il a défait ?)
Le Houérou H.-N., 2000. Restoration and rehabilitation of arid and semiarid Mediterranean ecosystems in North Africa and West Asia: a review. Arid Soil Res. Rehabil., 14 (1): 3-14, doi: 10.1080/089030600263139

Le Houérou H.-N., 2006. Agroforestry and sylvopastoralism: The role of trees and shrubs (Trubs) in range rehabilitation and development. Sécheresse, 17: $343-348$

Louhaichi M., Ghassali F., Salkini A.-K., Petersen S.-L., 2012. Effet de pâturage des moutons sur les communautés végétales de parcours : étude de cas de dépressions du paysage dans syriennes steppes arides. J. Arid Environ., 79: 101-106, doi: 10.1016/j.jaridenv.2011.11.024

MADR, 2007. Atelier international du Parlement panafricain sur la lutte contre la désertification, Alger, Algérie, 2-4 Avr. 2007. Ministère de I'Agriculture et du Développement rural, Alger, Algérie

Marnay L., Doligez P., Trillaud C., 2014. Les aliments - L'analyse chimique des fourrages. Equipédia

Nedjraoui D., Bédrani S., 2008. La désertification dans les steppes algériennes : causes, impacts et actions de lutte. VertigO, 8 (1), doi : 10.4000/ vertigo. 5375

Quézel P., Santa S., 1962. Nouvelle flore de l'Algérie et des régions désertiques méridionales. CNES, Paris, France, $1170 \mathrm{p}$.

Rekik F., Bentouati A., Aidoud A., 2014. Evaluation des potentialités fourragères d'un parcours steppique à dominance de Salsola vermiculata L. dans I'est de l'Algérie. Livest. Res. Rural Dev., 26, 231

Salemkour N., Aidoud A., Chalabi K., Chefrour A., 2016. Evaluation des effets du contrôle de pâturage dans des parcours steppiques arides en Algérie. Rev. Ecol. Terre Vie, 71 (2) : 178-191

Yerou H., 2013. Dynamique des systèmes d'élevage et leur impact sur l'écosystème steppique : cas de la région de Naâma (Algérie occidentale). Thèse Doct., Université Abou Bakr Belkaid, Tlemcen, Algérie, 135 p.

\section{Summary}

Amrani O., Chehma A.E. Plantation of Atriplex canescens for the restoration of degraded courses in the Algerian steppe

The Algerian steppe is the breeding environment of sheep, which graze on the rangelands. In its arid part, the vulnerability of the environment has been aggravated by this main use, which has intensified in the last decades. A decrease in fodder productivity characterizes this regressive trend, and perennial species are the most affected. The Algerian State has implemented a program to manage highly degraded steppe areas by planting resistant and productive biomass plant species such as Atriplex spp. The objective of this work was to estimate the pastoral productivity of a few rehabilitated stations by planting Atriplex canescens, compared to natural rangelands. The results showed an improvement in the vegetation cover (from $49 \%$ to $60 \%$ ) and better grazing values (phytomass, productivity, energy, and stocking). On the rehabilitated sites, energy and protein productivity of 604 milk forage units / ha/year and $29 \mathrm{~kg}$ dry matter / ha/year, and stocking of 1.51 sheep unit / ha/year were recorded, whereas on the degraded rangelands these values were $14 \mathrm{MFU}, 0.422 \mathrm{~kg}$ DM/ha/year, and 0.03 sheep unit / ha/year, respectively. The natural stations are overgrazed, and the vegetation and steppe soils have been degrading considerably. Unless steppe rangelands are restored or rehabilitated, their capacity for pastoral productivity will be severely reduced.

Keywords: Atriplex canescens, steppes, pastoral lands, sustainable land management, biomass, chemical composition, overgrazing, Algeria

\section{Resumen}

Amrani O., Chehma A.E. Plantaciones de Atriplex canescens con el fin de restaurar los senderos degradados en la estepa argelina

La estepa argelina es el medio de crianza de la oveja mediante la explotación de sus senderos. En esta parte árida, la vulnerabilidad del medio se ve agravada por una explotación principalmente pastoril, más y más intensa durante estas últimas décadas. Esta tendencia regresiva se caracteriza por una disminución de la productividad forrajera y las especies perennes son las más afectadas. El estado argelino a puesto en obra un programa de recuperación de los espacios de la estepa más degradados, a través del cultivo de especies vegetales resistentes y productivas de biomasa, como Atriplex spp. El objetivo de este estudio fue el de estimar la productividad pastoril de algunas estaciones rehabilitadas mediante el cultivo de Atriplex canescens, en comparación a los senderos naturales pastoreados. Los resultados muestran un mejoramiento de la cobertura vegetal $(49 \%$ a $60 \%)$ y mejores valores pastoriles (fitomasa, productividad, valor energético y carga pastoril). En las zonas rehabilitadas se registró una productividad energética y proteica de 604 unidades forrajeras leche/ha/año y $29 \mathrm{~kg}$ de materia seca / ha/ año, y una carga pastoril de 1,51 unidad ovina/ha/año, mientras que en los senderos naturales degradados estos valores fueron de 14 UFL, 0,422 $\mathrm{kg} \mathrm{MS} / \mathrm{ha} / \mathrm{año}$, et 0,03 unidad ovina/ha/año respectivamente. Las estaciones naturales son sobre explotadas y tienden hacia un estado de degradación severo de la vegetación y de los suelos de la estepa. A menos que se restauren o rehabiliten los senderos de la estepa, su capacidad de productividad pastoril se verá fuertemente reducida.

Palabras clave: Atriplex canescens, estepas, tierras de pastoreo, ordenación de tierras sostenible, biomasa, composición química, sobrepastoreo, Argelia 
